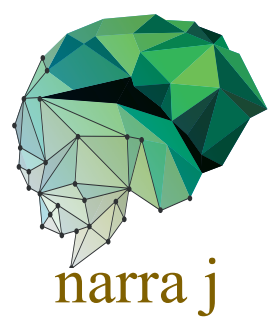

Short Communication

\title{
Attitude towards Zika among frontline physicians in a dengue-endemic country: A preliminary cross-sectional study in Indonesia
}

\begin{abstract}
Amanda Yufika1,2, Samsul Anwar3, Reza Maulana1, Nur Wahyuniati', Rizki R. Ramadana', Ikram Ikram1, Mudatsir Mudatsir1,4, Prattama S. Utomo5, Haypheng Te6,7, Seyi Samson Enitan8, Salin Sirinam9, Ruth Müller1o,11 and Abdul M. Setiawan12*

${ }^{1}$ Medical Research Unit, School of Medicine, Universitas Syiah Kuala, Banda Aceh, Indonesia; ${ }^{2}$ Department of Family Medicine, School of Medicine, Universitas Syiah Kuala, Banda Aceh, Indonesia; 3Department of Statistics, Faculty of Mathematics and Natural Sciences, Universitas Syiah Kuala, Banda Aceh, Indonesia; 4Department of Microbiology, School of Medicine, Universitas Syiah Kuala, Banda Aceh, Indonesia; 5Department of Medical Education, Faculty of Medicine, Gadjah Mada University, Jogjakarta, Indonesia; ${ }^{\circ S i e m ~ R e a p ~ P r o v i n c i a l ~ H o s p i t a l, ~ S i e m ~ R e a p, ~ C a m b o d i a ; ~} 7$ Siem Reap Provincial Health Department, Ministry of Health, Cambodia; ${ }^{8}$ Department of Medical Laboratory Science, Babcock University, Ilishan-Remo, Nigeria; 9 Department of Tropical Pediatrics, Faculty of Tropical Medicine, Mahidol University, Bangkok, Thailand; 10Institute of Occupational Medicine, Social Medicine and Environmental Medicine, Goethe University, Frankfurt am Main, Germany; ${ }^{11}$ Unit of Medical Entomology, Institute of Tropical Medicine, Antwerp, Belgium; ${ }^{12}$ Department of Microbiology, Faculty of Medicine and Health Sciences, Maulana Malik Ibrahim State Islamic University of Malang, Malang, Indonesia
\end{abstract}

*Corresponding author: abdul.malik.setiawan@kedokteran.uin-malang.ac.id

\section{Abstract}

In dengue-endemic countries such as Indonesia, Zika may be misdiagnosed as dengue, leading to underestimates of Zika disease and less foreknowledge of pregnancy-related complications such as microcephaly. Objective: To assess the attitudes of frontline physicians in a dengue-endemic country toward testing for Zika infection among patients with dengue-like illnesses. Methods: A cross-sectional online survey was conducted among general practitioners (GPs) in Indonesia. The survey assessed their attitude and also collected sociodemographic data, characteristics of their medical education, professional background, and workplace, and exposure to Zika cases. A two-step logistic regression analysis was used to assess possible variables associated with these attitudes. Results: A total of 370 GPs were included in the final analysis of which $70.8 \%$ had good attitude. Unadjusted analyses suggested that GPs who were 30 years old or older and those who had medical experience five years or longer had lower odds of having a positive attitude compared to those who aged younger than 30 years and those who had medical experience less than five years, OR: 0.58 ; 95\%CI: 0.37 , 0.91 and OR: 0.55 ; 95\%CI: 0.35 , 0.86 , respectively. No explanatory variable was associated with attitude in the fully adjusted model. Conclusion: Our findings point to younger GPs with a shorter medical experience being more likely to consider testing for Zika infection among their patients presenting with dengue-like illnesses. Strategic initiatives may be needed to enhance older or longer-experienced physicians' capacity in diagnosing Zika infection.

Keywords: Zika virus, Zika fever, attitude, general practitioner, Indonesia

\section{Introduction}

A pproximately 3.9 billion people in more than 120 different countries are at risk of becoming infected with three major arboviruses: dengue virus (DENV), chikungunya virus (CHIKV), and 
Zika virus (ZIKV) [1]. Globally, dengue incidence has increased 30-fold in the past 50 years [2]. Since it was first reported in the country in 1968 in the capital of Jakarta, dengue has become the most important arboviral disease in Indonesia with significant morbidity, mortality, and economic consequences [3-5]. Although no outbreak of Zika have yet been reported, serology [6] and molecular virology studies [7] have provided evidence that ZIKV is present in Indonesia, superimposed upon the burden of existing arboviruses in the country. It has been hypothesized that background herd immunity to ZIKV or cross-protection by antibodies against other flaviviruses such as DENV might have prevented the development of large ZIKV outbreaks in Asian countries including Indonesia [8].

Due to antigenic similarity, similar clinical presentations and the lack of a widely available specific Zika diagnostic tests, co-circulation of both DENV and ZIKV could lead to potential misclassification of diagnoses and an underestimate of Zika cases. This could increase the risk of pregnancy-related complications that are specific to ZIKV, but not DENV, infection. Early diagnosis of Zika could help mitigate severe pregnancy-related complications. However, there is a lack of understanding the attitude of frontline physicians towards initiating the processes to diagnose Zika in a dengue-endemic country like Indonesia. Therefore, the aim of this study was to assess the attitudes towards Zika testing of frontline physicians in a dengue-endemic country. Information generated from this study will better understand clinical readiness and vigilance of physicians against future outbreaks of Zika.

\section{Materials and Methods}

\section{Study design and setting}

A cross-sectional online survey was conducted among general practitioners (GPs) in Indonesia. Invitations to complete an anonymous online survey were distributed by social media to the members of doctor organizations or groups. Prior to the start of the survey, an introduction page showed information on investigators and contact details, aims of the study, and its expected benefits. The survey was estimated to take approximately 10 minutes.

\section{Study instrument and variables}

We developed the questionnaire to assess the attitude and to collect a range of potential explanatory variables. The questionnaire was developed in Bahasa Indonesia and was tested for its reliability among 30 participants during a pilot study. Two medical microbiologists were appointed to evaluate the questionnaire's validity and the questionnaire was finalized using feedback gained in the pilot test.

The response variable in this study was attitudes toward testing for Zika infection among patients with dengue-like illness. To assess the attitude, participants were provided with the following three statements: (Q1) "Patients with dengue-like illness (presenting with dengue signs and symptoms) might possibly have been infected with Zika"; (Q2) "Patients with dengue-like illness with a history of visiting Zika endemic countries should be suspected for Zika infection"; and (Q3) "Patients with dengue-like illness with a history of visiting Zika endemic countries, should be recommended to be tested for Zika test, if a lab facility is available" (Q3). The possible answers were on a five-point Likert scale. Responses of "Strongly agree" and "Agree" were classified as a positive attitude while "Strongly disagree", "Disagree" and "Neither agree nor disagree" were classified as a negative attitude. Participants were dichotomized into having a positive attitude if they had positive attitude for all three questions.

The explanatory variables were sociodemographic factors, characteristics of their medical education, professional background, and workplace, and exposure to Zika cases. Basic demographic factors included gender, age [classified into less than 30-years old and 30-years old or older], and educational attainment [medical doctor and medical doctor with master degree]. Type of occupation was divided into GPs, GPs who also had faculty appointments, and GPs who were also specialist residents. To collect their workplace characteristics, the participants were asked about the type of workplace [community health center (known as Pusat Kesehatan Masyarakat [Puskesmas]), private clinic or hospital and public hospital] and location of workplace [district (rural), regency (sub-urban) and province (urban)]. In addition, the participants were asked whether they have attended local, national or international conference in 
the last five months as well as their length of medical practice experience in year. To assess their workplace facility, the participants were asked the availability of specific testing procedures such as polymerase chain reaction (PCR) and enzyme-linked immunosorbent assay (ELISA) and the availability of access to scientific journals. To assess the exposure to Zika cases, the participants were also asked whether they had contact with patient(s) presenting signs and symptoms of ZIKV infection in a clinical setting.

\section{Statistical analysis}

To assess the possible variables associated with positive attitude, a two-step logistic regression analysis was employed. In the unadjusted analysis, all explanatory variables were analyzed separately. Variables with $\mathrm{p} \leq 0.25$ in this step were entered into the adjusted analysis. Estimated unadjusted odds ratio (OR) and adjusted OR (aOR) were interpreted in relation to a reference category. For all analyses, significance was assessed at $\alpha=0.05$.

\section{Results}

\section{Demographic characteristics}

We included 370 participants in the final analysis; of these, 41.1\% (152) were male and 223 (60.3\%) aged younger than 30 years old (Table 1). Less than $10 \%$ of participants had an additional master degree and $13.5 \%$ of the participants were also working as faculty in a university. Approximately 22\% of GPs were working in the Puskesmas and approximately equal proportions were working at the levels of district, regency and province. Although $67 \%$ of participants had attended a province-level conference in the last five months, only $5.4 \%$ had attended an international-level conference. In term of workplace facilities, $85.7 \%$ and $83.0 \%$ of respondents stated that they were working in healthcare centers that had no PCR and ELISA facility, respectively. Only 26 (7.0\%) of the participants had had contact with patients presenting signs and symptoms compatible with ZIKV infection prior to the survey.

\section{Attitude to initiate Zika infection and associated explanatory variables}

There were 280 (75.6\%), 335 (90.5\%) and 334 (90.2\%) who had positive attitude for Q1, Q2 and Q3, respectively. Data indicated that most participants $(70.8 \%, 262)$ had a good attitude towards Zika testing among patients with dengue-like illness. In unadjusted analyses, age group and medical experience were associated with attitude. Those who aged 30 years old or older had lower odds of having positive attitude compared to those who aged younger than 30 years with OR: 0.58; 95\%CI: 0.37-0.91 (Table 1). Participants who had medical experience five years or longer were also associated lower odds of having positive attitude compared to those who had shorter medical experience, OR: 0.55; 95\%CI: 0.35-0.86. In the adjusted analysis model, no explanatory variable was associated with GP attitude (Table 1).

\section{Discussion}

DENV and ZIKV are closely related flaviviruses with similar transmission cycles and disease manifestations. The diagnostics of Zika is particularly confusing in dengue-endemic countries because of similar symptoms and cross-reactivity with dengue. Front-line physicians considering Zika infection among those with dengue-like illness are critical to early diagnosis of Zika and mitigation of severe pregnancy-related complications. The present study found that more than $70 \%$ of the frontline physicians knew that patients with dengue-like illness might have ZIKV infection; those with a history of visiting Zika endemic countries should be suspected of Zika infection and therefore should be recommended to be tested for Zika. This number is higher compared to previous findings regarding attitudes towards Zika-related issues [9, 10]. Altogether, these studies suggesting that attitudes towards Zika are influenced by the nature of the issue; the attitude towards general issue of Zika is lower [9] compared to attitude towards pregnancyrelated complications of Zika infection [10]. 
Table 1. Unadjusted and adjusted logistic regression analyses showing predictors of positive attitude towards Zika diagnostic testing ( $\mathrm{n}=370$ )

\begin{tabular}{|c|c|c|c|c|c|c|}
\hline \multirow[t]{2}{*}{ Variable } & \multirow[t]{2}{*}{$\mathrm{n}(\%)$} & \multirow{2}{*}{$\begin{array}{l}\text { Attitude } \\
\text { Positive (\%) }\end{array}$} & \multicolumn{2}{|l|}{ Unadjusted } & \multicolumn{2}{|l|}{ Adjusted } \\
\hline & & & OR (95\% CI) & $p$-value & OR (95\% CI) & $p$-value \\
\hline \multicolumn{7}{|l|}{ Gender } \\
\hline Male $(R)$ & $152(41.1)$ & $105(69.1)$ & 1 & & & \\
\hline Female & $218(58.9)$ & $157(72.0)$ & $1.15(0.73,1.81)$ & 0.541 & - & - \\
\hline \multicolumn{7}{|l|}{ Age group (year) } \\
\hline$<30(R)$ & $223(60.3)$ & $168(75 \cdot 3)$ & 1 & & 1 & \\
\hline 30 or more & $147(39.7)$ & $94(63.9)$ & $0.58(0.37,0.91)$ & 0.019 & $0.80(0.39,1.65)$ & 0.547 \\
\hline \multicolumn{7}{|l|}{ Educational attainment } \\
\hline $\mathrm{GP}(R)$ & $337(91.1)$ & $241(71.5)$ & 1 & & & \\
\hline GP with master degree & $33(8.9)$ & $21(63.6)$ & $0.70(0.33,1.47)$ & 0.344 & - & - \\
\hline \multicolumn{7}{|l|}{ Type of occupation } \\
\hline GP $(R)$ & $287(77.6)$ & $209(72.8)$ & 1 & & 1 & \\
\hline GP and faculty & $50(13.5)$ & $34(68.0)$ & $0.79(0.42,1.52)$ & 0.484 & $1.06(0.52,2.14)$ & 0.876 \\
\hline Residency & $33(8.9)$ & $19(57.6)$ & $0.51(0.24,1.06)$ & 0.071 & $0.79(0.34,1.83)$ & 0.582 \\
\hline \multicolumn{7}{|l|}{ Type of workplace } \\
\hline Community health center $(R)$ & $84(22.7)$ & $60(71.4)$ & 1 & & & \\
\hline Private clinic or hospital & $150(40.5)$ & $106(70.7)$ & $0.96(0.53,1.74)$ & 0.902 & - & - \\
\hline Public hospital & $136(36.8)$ & $96(70.6)$ & $0.96(0.53,1.75)$ & 0.894 & - & - \\
\hline \multicolumn{7}{|l|}{ Location of workplace } \\
\hline District $(R)$ & $113(30.5)$ & $82(72.6)$ & 1 & & & \\
\hline Regency & $140(37.8)$ & $99(70.7)$ & $0.91(0.53,1.58)$ & 0.746 & - & - \\
\hline Province & $117(31.6)$ & $81(69.2)$ & $0.85(0.48,1.50)$ & 0.578 & - & - \\
\hline \multicolumn{7}{|l|}{ Attended a province-level conference } \\
\hline No $(R)$ & $122(33.0)$ & $87(71.3)$ & 1 & & & \\
\hline Yes & $248(67.0)$ & $175(70.6)$ & $0.96(0.60,1.56)$ & 0.882 & - & - \\
\hline \multicolumn{7}{|l|}{ Attended a national-level conference } \\
\hline No $(R)$ & $254(68.6)$ & $185(72.8)$ & 1 & & 1 & \\
\hline Yes & $116(31.4)$ & $77(66.4)$ & $0.74(0.46,1.18)$ & 0.206 & $0.79(0.49,1.29)$ & 0.343 \\
\hline \multicolumn{7}{|c|}{ Attended an international-level conference } \\
\hline No $(R)$ & $350(94.6)$ & $250(71.4)$ & 1 & & & \\
\hline Yes & $20(5.4)$ & $12(60.0)$ & $0.60(0.24,1.51)$ & 0.279 & - & - \\
\hline \multicolumn{7}{|l|}{ Medical experience } \\
\hline Less than 5 years $(R)$ & $245(66.2)$ & $184(75.1)$ & 1 & & 1 & \\
\hline 5 or more & $125(33.8)$ & $78(62.4)$ & $0.55(0.35,0.86)$ & 0.012 & $0.70(0.33,1.48)$ & 0.346 \\
\hline \multicolumn{7}{|l|}{ Workplace has PCR facility } \\
\hline No $(R)$ & $317(85.7)$ & $224(70.7)$ & 1 & & & \\
\hline Yes & $53(14.3)$ & $38(71.7)$ & $1.05(0.55,2.00)$ & 0.878 & - & - \\
\hline \multicolumn{7}{|l|}{ Workplace has ELISA facility } \\
\hline No $(R)$ & $307(83.0)$ & $216(70.4)$ & 1 & & & \\
\hline Yes & $63(17.0)$ & $46(73.0)$ & $1.14(0.62,2.09)$ & 0.673 & - & - \\
\hline Workplace has access to journals & & & & & & \\
\hline
\end{tabular}



No $(R)$
Yes
Had contact with patients with Zika signs and symptoms
No $(R)$

$285(77.0)$

$85(23.0)$

344 (93.0) $26(7.0)$

CI: confidence interval, OR: odds ratio, R: reference group
$205(71.9)$

$57(67.1)$

244 (70.9) $18(69.2)$

0.387

$0.79(0.47,1.34)$ $0.92(0.39,2.19)$

0.854 
Our study found that, in an unadjusted analysis, younger GPs and who had shorter years of medical experience had better attitude compared to their senior colleagues who had longer medical experiences. This finding is supported by a previous study [11] explaining that Zika infection is a new re-emerging infectious disease and most information is available on online media. Younger GPs are more active in accessing online media compared to their senior counterparts. ZIKV has the potential to cause severe pregnancy-related complications, including microcephaly, and so it will be important to convey the importance of this disease to senior frontline healthcare providers.

Our study found that only $14.3 \%$ and $17.0 \%$ of respondents were working in workplaces where PCR and ELISA facility were available, respectively. This suggests that most of the respondents were working in in workplaces that had no diagnostic capacity on Zika infection. Although diagnostic capacity was expected to influence participants `attitude towards Zika testing, our data suggested that this workplace facility has no association with attitude.

This study might have some limitations. There are potential biases in geographical selection due to internet connection disparities; regions with less-developed internet infrastructures might be less well represented in this study. The limited number of samples in this study might restrict this study's generalizability to a national scope.

\section{Conclusion}

Attitude towards initiation of early diagnose of ZIKV infection among frontline physician is relatively high, however, the present study suggests that strategies for enhancing the capacity of medical doctors, and especially of old-fashioned physicians in Indonesia may be needed.

\section{Declarations}

\section{Ethics approval}

The protocol used in this study was approved by the Ethical Clearance Committee of the School of Medicine, Universitas Syiah Kuala University, Banda Aceh, Indonesia. Informed consent was obtained from all participants prior to their participation in the survey.

\section{Acknowledgments}

We thank the respondents of this study for their willingness to participate.

\section{Conflict of interest}

The authors declared no potential conflicts of interest with respect to the research, authorship, and/or publication of this article.

\section{Funding}

This study received no funding.

\section{How to cite}

Yufika A, Anwar S, Maulana R, et al. Attitude towards Zika among frontline physicians in a dengue-endemic country: A cross-sectional study in Indonesia. Narra J 2021; 1(1): e32 http://doi.org/10.52225/narraj.v1i1.e32.

\section{References}

1. Shragai T, Tesla B, Murdock C, et al. Zika and chikungunya: Mosquito-borne viruses in a changing world. Ann N Y Acad Sci 2017; 1399(1):61-77

2. Guzman MG, Harris E. Dengue. Lancet 2015; 385(9966):453-465. 
Yufika et al. Narra J 2021; 1(1): e32 - http://doi.org/10.52225/narraj.v1i1.e32

3. Harapan H, Michie A, Yohan B, et al. Dengue viruses circulating in Indonesia: A systematic review and phylogenetic analysis of data from five decades. Rev Med Virol 2019; 29(4):e2037.

4. Harapan H, Michie A, Mudatsir M, et al. Epidemiology of dengue hemorrhagic fever in Indonesia: Analysis of five decades data from the National Disease Surveillance. BMC Res Notes 2019; 12(1):350.

5. Dhewantara P, Jamil K, Fajar J, et al. Decline of notified dengue infections in Indonesia in 2017: Discussion of the possible determinants. Narra J 2021; 1(1):e23.

6. Sasmono RT, Dhenni R, Yohan B, et al. Zika sirus seropositivity in 1-4-year-old children, Indonesia, 2014. Emerg Infect Dis 2018; 24(9);1740-1743.

7. Perkasa A, Yudhaputri F, Haryanto S, et al. Isolation of Zika virus from febrile patient, Indonesia. Emerg Infect Dis 2016; 22(5):924-925.

8. Musso D, Lanteri MC. Zika virus in Singapore: Unanswered questions. Lancet Infect Dis 2017; 17(8):782-783.

9. Harapan H, Alleta A, Anwar S, et al. Attitudes towards Zika virus infection among medical doctors in Aceh province, Indonesia. J Infect Public Health 2018; 11(1):99-104.

10. Harapan H, Rajamoorthy Y, Utomo PS, et al. Knowledge and attitude towards pregnancy-related issues of Zika virus infection among general practitioners in Indonesia. BMC Infect Dis 2019; 19(1):693.

11. Harapan H, Aletta A, Anwar S, et al. Healthcare workers knowledge towards Zika virus infection in Indonesia: A survey in Aceh. Asian Pac J Trop Med 2017; 10(2):189-194. 